\title{
Almost sure stability of the delayed Markovian jump RDNNs
}

\author{
Weiyuan Zhang ${ }^{1 *}$ and Junmin $\mathrm{Li}^{2}$
}

\author{
"Correspondence: ahzwy@163.com \\ ${ }^{1}$ Institute of Nonlinear Science, \\ Xianyang Normal University, \\ Xianyang, P.R. China \\ Full list of author information is \\ available at the end of the article
}

\begin{abstract}
In this paper, we consider the almost sure stability of the delayed reaction-diffusion neural networks (RDNNs) with Markovian jump parameters and Dirichlet boundary conditions. By constructing new Lyapunov functional and utilizing some inequality techniques we give sufficient conditions ensuring the almost sure stability. The criteria can also ensure the almost sure global exponential stability when the input is equal to zero. Two numerical examples are given to demonstrate the effectiveness of the proposed approach.
\end{abstract}

Keywords: Reaction-diffusion neural networks; Almost sure stability; Delayed; Markovian jump

\section{Introduction}

During the past few decades, neural networks(NNs)have been successfully applied in many areas such as signal processing, image processing, pattern recognition, fault diagnosis, associative memory, and combinatorial optimization [1-3]. Sontag [4] firstly introduced the concept of input-to-state stability (ISS). The theory of ISS plays a central role in modern control theory, in particular, in robust stabilization of nonlinear systems, design of nonlinear observers, analysis of large-scale networks, etc. [4-9]. Roughly speaking, the ISS property implies that no matter what the initial state is, if the external input is small, then the state must be eventually small. In last years, many interesting results on ISS properties of various systems such as discrete systems, switched systems, and hybrid systems have been reported [10-12]. Since the ISS property implies that not only the unperturbed system is asymptotically stable at the equilibrium for the unforced system, but also its behavior remains bounded when its inputs are bounded. It also offers an effective way to tackle the stabilization of nonlinear control in the presence of various uncertainties arising from observer design, new small-gain theorems, and control engineering applications [7-9]. Because of these research backgrounds, the ISS properties of NNs are considered in recent years. It is well known that NNs are often affected by noise, such as perturbations in control or errors on observation. Thus, NNs are required not only to be stable, but also to have the property of ISS. Therefore, finding sufficient conditions to guarantee the ISS of NNs is an important and meaningful research topic. A great number of results on this topic have appeared in the literature [13-17]. For instance, Sanchez and Perez [13] firstly proposed the ISS properties and presented some matrix norm conditions on ISS for NNs. Ahn [14] considered a passivity-based learning law to investigate the ISS for a class of

(c) The Author(s) 2018. This article is distributed under the terms of the Creative Commons Attribution 4.0 International License (http://creativecommons.org/licenses/by/4.0/), which permits unrestricted use, distribution, and reproduction in any medium, provided you give appropriate credit to the original author(s) and the source, provide a link to the Creative Commons license, and indicate if changes were made. 
switched Hopfield neural networks with time delay. Some LMI sufficient conditions have been proposed to guarantee the ISS by using Lyapunov function method [16]. In [17], two new criteria on ISS of NNs with time-varying delays are given.

Many pattern formation and wave propagation phenomena that appear in nature can be described by systems of coupled nonlinear differential equations, generally known as reaction-diffusion equations [18]. These wave propagation phenomena are exhibited by systems belonging to very different scientific disciplines. Therefore, the reaction-diffusion effects cannot be neglected in both biological and man-made NNs, especially when electrons are moving in a noneven electromagnetic field. So we must consider that the activations vary in space and time. Recently, the stability or synchronization criteria for NNs involving diffusion and time-varying delays are found in [18-31]. Moreover, note that many systems such as biological networks and man-made NNs are described by partial differential equations with Dirichlet boundary conditions [19, 21, 23, 32], instead of Neumann boundary conditions. For example, in [29], the global exponential synchronization stability in an array of linearly diffusively coupled delayed RDNNs was studied via adding an impulsive controller to a small fraction of nodes. In [30], the authors discussed the sampled-data synchronization for a class of RDNNs with Dirichlet boundary conditions. Unlike other studies, a sampled-data controller with stochastic sampling is designed to synchronize the concerned delayed RDNNs. In [31], the global asymptotic sampled-data synchronization problem of an array of $N$ randomly coupled RDNNs with Markovian jumping parameters and mixed delays was investigated. The jump parameters are determined by a continuous-time discrete-state Markovian chain, and the mixed time delays under consideration comprise both discrete and distributed delays. Hence, it is necessary to study ISS of reaction-diffusion delayed NNs with Dirichlet boundary conditions.

Over the past few years, stability analysis for stochastic systems with Markovian jump parameters or Brownian motion defined in a complete probability space or stochastic disturbances was widely investigated [31, 33-37]. Markovian jump systems can be described by a set of linear systems with the transitions among models determined by a Markovian chain in a finite mode set. Applications of this kind of systems can be found in economic systems, power systems, solar-powered systems, battle management in command, control and communication systems, etc. NNs in real life often have a phenomenon of information latching. It is recognized that a way for dealing with this information latching problem is to extract finite-state representations (also called modes or clusters). In fact, such NNs with information latching may have finite modes, the modes may switch (or jump) from one to another at different times, and the switching (or jumping) between two arbitrarily different modes can be governed by a Markovian chain. Hence, the NNs with Markovian jump parameters are of great significance in modeling a class of NNs with finite modes. Recently, some results on the stability, estimation, and control problems related to such systems have been reported in the literature [35-40].

To the best of author's knowledge, there are few results or even no results concerning the ISS issues for Markovian jump RDNNs with mixed time-varying delays and Dirichlet boundary conditions. The issues of integrating mixed time-varying delays, Markovian jump parameters and diffusion effects into the study of almost sure ISS for NNs require more complicated analysis, which is very important in both theory and applications. In the present paper, we give some sufficient conditions for almost sure ISS for RDNNs with 
mixed delays and Markovian jump parameters. It is a challenging and interesting problem how to develop Lyapunov methods to solve the ISS problem of mixed delayed stochastic RDNNs with Markovian jump parameters. As far as we know, this extension has not been reported in the literature at the present stage. Compared with the existing results, the main contributions of this paper can be summarized as follows: the first involves that we make the first attempt to address the ISS analysis for a class of RDNNs with mixed delays and Markovian jump parameters; the second relates to that we apply the well-known Hardy-Poincaré inequality and Lyapunov method to investigate ISS properties for mixed delayed stochastic RDNNs with Markovian jump parameters; the third aspect is that the established algebraic criteria for ISS of such a system are new in terms of mixed delays, Markovian jump parameters, and reaction-diffusion terms. We conclude that both the reaction-diffusion coefficients and the regional feature have an effect on the almost sure ISS. The provided ISS criteria are true to Dirichlet boundary conditions and concerned with the regional feature, the reaction-diffusion coefficients, and the first eigenvalue of the Dirichlet Laplacian. Finally, two examples are employed to demonstrate the usefulness of the obtained results.

The structure of this paper is outlined as follows. In Sect. 2, we introduce some preliminaries and lemmas. In Sect. 3, we state the main results. In Sect. 4, we present two numerical examples to illustrate the results and, finally, make some conclusions in Sect. 5 .

\section{Model description and preliminaries}

To begin with, we introduce some notations. Let $\Omega$ is an open domain containing the origin and radially bounded by $\pi$, with smooth boundary $\partial \Omega$ and mes $\Omega>0$ in space $R^{m}$, where mes $\Omega$ is the measure of the set $\Omega$. By $L^{2}(\Omega)$ we denote the space of real Lebesguemeasurable functions defined on $\Omega$; it is a Banach space with the $L_{2}$-norm $\|\eta(t, x)\|_{2}=$ $\left[\sum_{i=1}^{n}\left\|\eta_{i}(t, x)\right\|_{2}^{2}\right]^{\frac{1}{2}}$, where $\eta(t, x)=\left(\eta_{1}(t, x), \ldots, \eta_{n}(t, x)\right)^{T},\left\|\eta_{i}(t, x)\right\|_{2}=\left(\int_{\Omega}\left|\eta_{i}(t, x)\right|^{2} d x\right)^{1 / 2}$, $|\cdot|$ denotes the absolute value. By $C=C\left((-\infty, 0] \times \Omega, R^{n}\right)$ we denote the Banach space of continuous functions mapping the set $(-\infty, 0] \times \Omega$ into $R^{n}$ with the norm $\|\varphi\|=$ $\sqrt{\sum_{i=1}^{n}\left\|\varphi_{i}\right\|_{2}^{2}}$, where $\varphi(t, x)=\left(\varphi_{1}(t, x), \ldots, \varphi_{n}(t, x)\right)^{T},\left\|\varphi_{i}\right\|_{2}=\sqrt{\int_{\Omega}\left|\varphi_{i}(\cdot, x)\right|_{\tau}^{2} d x},\left|\varphi_{i}(\cdot, x)\right|_{\tau}=$ $\sup _{-\infty<s \leq 0}\left|\varphi_{i}(s, x)\right|$. Let $\left(\Omega, F,\left\{F_{t}\right\}_{t \geq 0}, P\right)$ be a complete probability space with filtration $\left\{F_{t}\right\}_{t \geq 0}$ satisfying the usual conditions (i.e., the filtration contains all $P$-null sets and is right continuous). By $L_{F_{0}}^{p}\left((-\infty, 0] \times \Omega ; R^{n}\right)$ we denote the family of all $F_{0}$-measurable $C\left((-\infty, 0] \times \Omega ; R^{n}\right)$-valued random variables $\varphi=\{\varphi(s, x):-\infty<s \leq 0, x \in \Omega\}$ such that $E\|\varphi\|_{2}^{2}<+\infty$, where $E\{\cdot\}$ stands for the mathematical expectation operator with respect to the given probability measure $P$. By $\kappa$ we denote the class of continuous strictly increasing functions $\mu$ from $R^{+}$to $R^{+}$with $\mu(0)=0$. Let $\kappa_{\infty}$ denote the class of functions $\mu \in \kappa$ with $\mu(r) \rightarrow \infty$ as $r \rightarrow \infty$. Functions in $\kappa$ and $\kappa_{\infty}$ are called class $\kappa$ and $\kappa_{\infty}$ functions, respectively. In this note, a function $\beta: R^{+} \times R^{+} \rightarrow R^{+}$is said to be of class $\kappa L$ if, for each fixed $t$, the mapping $\beta(\cdot, t)$ is of class $\kappa$ and, for each fixed $s$, the function $\beta(s, t)$ is decreasing to zero in $t$ as $t \rightarrow \infty$. By $L_{\infty}^{n}(\Omega)$ we denote the class of functions $v(t, x): R^{+} \times \Omega \rightarrow R^{+}$ with the supremum norm $\|v(t, x)\|_{\Omega}=\sup _{t \geq 0}\|v(t, x)\|<\infty$. 
Consider the following delayed RDNNs with Markovian jump parameters:

$$
\begin{aligned}
\frac{\partial u_{i}(t, x)}{\partial t}= & \sum_{l=1}^{m} \frac{\partial}{\partial x_{l}}\left(D_{i l} \frac{\partial u_{i}(t, x)}{\partial x_{l}}\right)-a_{i}(r(t)) u_{i}(t, x)+\sum_{j=1}^{n} w_{i j}(r(t)) g_{j}\left(u_{j}(t, x)\right) \\
& +\sum_{j=1}^{n} h_{i j}(r(t)) g_{j}\left(u_{j}\left(t-\tau_{j}(t), x\right)\right)+\sum_{j=1}^{n} b_{i j}(r(t)) \int_{-\infty}^{t} k_{i j}(t-s) g_{j}\left(u_{j}(s, x)\right) d s \\
& +v_{i}(t, x), \quad t \geq 0, x \in \Omega,
\end{aligned}
$$

where $x=\left(x_{1}, \ldots, x_{m}\right)^{T} \in \Omega, u_{i}(t, x)$ represents the state of the $i$ th neuron at time $t$ and in space $x$; the diagonal matrix $A(r(t))=\operatorname{diag}\left(a_{1}(r(t)), \ldots, a_{n}(r(t))\right)$ has positive entries $a_{i}(r(t))>0, B(r(t))=\left(b_{i j}(r(t))\right)_{n \times n}, W(r(t))=\left(w_{i j}(r(t))\right)_{n \times n}$, and $H(r(t))=\left(h_{i j}(r(t))\right)_{n \times n}$ are the interconnection matrices representing the weight coefficients of the neurons, $g_{j}$ denotes the activation functions of the $j$ th neuron at time $t$ and in space $x, v(t, x)=$ $\left(v_{1}(t, x), v_{2}(t, x), \ldots, v_{n}(t, x)\right)^{T}$ denotes an external input vector to neurons, $\tau_{j}(t)$ are timevarying delays of NNs satisfying $0 \leq \tau_{j}(t) \leq \tau$ and $\dot{\tau}_{j}(t) \leq \mu<1$, smooth functions $D_{i l}=$ $D_{i l}(t, x, u) \geq 0$ stand for the transmission diffusion operators along the $i$ th neuron, and $k_{i j}(\cdot)$ are delay kernels.

Let $\{r(t), t \geq 0\}$ be a right-continuous Markovian chain on the probability space taking values in the finite space $S=\{1,2, \ldots, N\}$ with generator $\Gamma=\left(\gamma_{i j}\right)_{N \times N}$ given by

$$
P\{r(t+\delta)=j \mid r(t)=i\}= \begin{cases}\gamma_{i j} \delta+o(\delta), & \text { if } i \neq j, \\ 1+\gamma_{i j} \delta+o(\delta), & \text { if } i=j,\end{cases}
$$

with $\delta>0$ and $\lim _{\delta \rightarrow 0} o(\delta) / \delta=0$, where $\gamma_{i j} \geq 0$ is the transition rate from $i$ to $j$ if $i \neq j$ and $\gamma_{i i}=-\sum_{i \neq j} \gamma_{i j}$. It is known that almost every sample path of $r(t)$ is a right-continuous step function with finite number of simple jumps in any finite subinterval of $R^{+}$.

System (1) is supplemented with the following Dirichlet boundary conditions and initial value:

$$
\begin{aligned}
& u_{i}(t, x)=0, \quad(t, x) \in[0,+\infty) \times \partial \Omega, \\
& u_{i}(s, x)=\varphi_{i}(s, x), \quad(s, x) \in(-\infty, 0] \times \Omega,
\end{aligned}
$$

where $\varphi(s, x)=\left(\varphi_{1}(s, x), \ldots, \varphi_{n}(s, x)\right)^{T}$ with given bounded and continuous functions $\varphi_{i}(s, x)$.

We denote $u_{i}(t, x)=u_{i}(t), \varphi_{i}(s, x)=\varphi_{i}(s), v_{i}(t, x)=v_{i}(t)$ if no confusion occurs.

To obtain our main results, we assume that the following conditions hold.

(A1) There exist positive constants $L_{j}$ such that, for all $\eta_{1}, \eta_{2} \in R$,

$$
0 \leq \frac{g_{j}\left(\eta_{1}\right)-g_{j}\left(\eta_{2}\right)}{\eta_{1}-\eta_{2}} \leq L_{j}
$$

(A2) The delay kernels $k_{i j}(\cdot):[0,+\infty) \rightarrow[0,+\infty)(i, j=1,2, \ldots, n)$ are real-valued nonnegative continuous functions that satisfy the following conditions:

(i) $\int_{0}^{+\infty} k_{i j}(s) d s=1$,

(ii) $\int_{0}^{+\infty} s k_{i j}(s) d s \leq+\infty$ for all $s \in[0,+\infty)$,

(iii) $\int_{0}^{+\infty} s k_{i j}(s) e^{\xi s} d s<+\infty$, where $\xi$ is a positive constant. 
Remark 1 By assumptions (A1) and (A2), when $v(t)$ is given, it is not difficult to prove that there exists a unique equilibrium point $u^{*}$ for system (1)-(2) based on Mawhin's continuation theorem [40].

Definition 1 System (1)-(2) is said to be input-to-state stable if there exist a class $\kappa L$ function $\beta$ and a class $\kappa$ function $\gamma$ such that, for any initial state $\varphi$ and any bounded input $v(t)$, the solution $u(t)$ exists for all $t \geq 0$ and satisfies

$$
E\|u(t)\|_{2} \leq \beta(E\|\varphi\|, t)+\gamma\left(\|v(t)\|_{\Omega}\right) .
$$

Remark 2 Inequality (3) guarantees that for any bounded inputs $v(t)$, the state $u(t)$ will be bounded. That is, if the delayed RDNNs are globally input-to-state stable, then the state of the delayed RDNNs should remain bounded when its inputs are bounded. Hence, the delayed RDNNs are bounded-input bounded-output stable.

Lemma 1 ([39, 41] Hardy-Poincaré inequality) Let $\Omega \subset R^{m}(m \geq 3)$ be a bounded open set containing the origin. Then

$$
\begin{aligned}
& \int_{\Omega}|\nabla u|^{2} d x-\frac{(m-2)^{2}}{4} \int_{\Omega} \frac{u^{2}}{|x|^{2}} d x \geq \frac{\Lambda_{2}}{R_{\Omega}^{2}} \int_{\Omega} u^{2} d S, \\
& u \in H_{0}^{1}(\Omega)=\left\{y\left|y \in L^{2}(\Omega), y\right|_{\partial \Omega}=0, D_{i} y=\frac{\partial y}{\partial x_{i}} \in L^{2}(\Omega), 1 \leq i \leq m\right\},
\end{aligned}
$$

$\Lambda_{2}=5.783 \ldots$ is the first eigenvalue of the Dirichlet Laplacian of the unit disk in $R^{2}$, and $R_{\Omega}$ is the radius of the ball $\Omega^{*} \subset R^{m}$ centered at the origin having the same measure as $\Omega$.

Remark 3 In this paper, we employ different inequalities to deal with the reactiondiffusion terms, and consequently we are convinced that the diffusion does contribute to the stability analysis of RDNNs. The Hardy-Poincaré inequality is an important result and has been widely utilized in the study of partial differential equation [39, 41]. The introduction of Lemma 1 is mostly for evaluating the reaction-diffusion terms. In [23, 27, 29], there is a similar estimate regarding the reaction-diffusion terms; meanwhile, $u \in C_{0}^{1}(\Omega)$ is required in $[23,27,29]$. Here we suppose that $u \in H_{0}^{1}(\Omega)$. Obviously, $u \in C_{0}^{1}(\Omega)$ is stronger than $u \in H_{0}^{1}(\Omega)$.

\section{Main results}

Theorem 1 Suppose that (A1)-(A2) hold. System (1)-(2) is almost sure ISS if there exist constants $q_{i}(i)>0$ for any $r(t)=i \in S, i, j=1,2, \ldots, n$, such that

$$
\begin{aligned}
-\Xi & -2 a_{i}(i)+2\left|w_{i i}(i)\right| L_{i}+\sum_{j=1, j \neq i}^{n}\left|w_{i j}(i)\right|+\sum_{j=1, j \neq i}^{n} \frac{q_{j}(i)}{q_{i}(i)}\left|w_{j i}(i)\right| L_{i}^{2}+\sum_{j=1}^{n}\left|h_{i j}(i)\right| L_{j}+\sum_{j=1}^{n}\left|b_{i j}(i)\right| \\
& +\sum_{j=1}^{n} \frac{q_{j}(i)}{q_{i}(i)}\left|b_{j i}(i)\right| L_{i}^{2}+\sum_{j=1}^{n} \frac{\left|h_{j i}(i)\right|}{1-\mu} e^{2 \alpha \tau} \frac{q_{j}(i)}{q_{i}(i)} L_{i}+\sum_{j=1}^{n} \gamma_{i j} q_{i}(j)+1 \\
<0 . &
\end{aligned}
$$

Here $\Xi=\frac{\alpha(m-2)^{2}}{2 \pi^{2}}+\frac{2 \alpha \Lambda_{2}}{R_{\Omega}^{2}}, \underline{\alpha}=\min \left\{D_{i l}, i=1, \ldots, n ; l=1, \ldots, m\right\}>0$, and $\pi$ is a radial bound of an open domain $\Omega$. 
Proof If condition (4) holds, then we can choose a positive number $\varepsilon$ (may be very small) such that, for $i=1,2, \ldots, n$,

$$
\begin{aligned}
-\Xi- & 2 a_{i}(i)+2\left|w_{i i}(i)\right| L_{i}+\sum_{j=1, j \neq i}^{n}\left|w_{i j}(i)\right|+\sum_{j=1, j \neq i}^{n} \frac{q_{j}(i)}{q_{i}(i)}\left|w_{j i}(i)\right| L_{i}^{2}+\sum_{j=1}^{n}\left|h_{i j}(i)\right| L_{j} \\
& +\sum_{j=1}^{n}\left|b_{i j}(i)\right|+\sum_{j=1}^{n} \frac{q_{j}(i)}{q_{i}(i)}\left|b_{j i}(i)\right| L_{i}^{2}+\sum_{j=1}^{n} \frac{\left|h_{j i}(i)\right|}{1-\mu} e^{2 \alpha \tau} \frac{q_{j}(i)}{q_{i}(i)} L_{i}+\sum_{j=1}^{n} \gamma_{i j} q_{i}(j)+1+\varepsilon
\end{aligned}
$$

$<0$.

Consider the following functions:

$$
\begin{aligned}
F_{i}\left(y_{i}\right)= & 2 y_{i}-\Xi-2 a_{i}(i)+2\left|w_{i i}(i)\right| L_{i}+\sum_{j=1, j \neq i}^{n}\left|w_{i j}(i)\right|+\sum_{j=1, j \neq i}^{n} \frac{q_{j}(i)}{q_{i}(i)}\left|w_{j i}(i)\right| L_{i}^{2}+\sum_{j=1}^{n}\left|h_{i j}(i)\right| L_{j} \\
& +\sum_{j=1}^{n}\left|b_{i j}(i)\right|+\sum_{j=1}^{n} \frac{q_{j}(i)}{q_{i}(i)}\left|b_{j i}(i)\right| L_{i}^{2} \int_{0}^{+\infty} k_{j i}(s) e^{2 y_{i} s} d s+\sum_{j=1}^{n} \frac{\left|h_{j i}(i)\right|}{1-\mu} e^{2 \alpha \tau} \frac{q_{j}(i)}{q_{i}(i)} L_{i} \\
& +\sum_{j=1}^{n} \gamma_{i j} q_{i}(j)+1 .
\end{aligned}
$$

From (6) and (A2) we obtain that $F_{i}(0)<-\varepsilon<0$ and $F_{i}\left(y_{i}\right)$ is continuous for $y_{i} \in[0,+\infty)$; moreover, $F_{i}\left(y_{i}\right) \rightarrow+\infty$ as $y_{i} \rightarrow+\infty$, and thus there exists constant $\alpha_{i} \in(0,+\infty)$ such that

$$
\begin{aligned}
F_{i}\left(\alpha_{i}\right)= & 2 \alpha_{i}-\Xi-2 a_{i}(i)+2\left|w_{i i}(i)\right| L_{i}+\sum_{j=1, j \neq i}^{n}\left|w_{i j}(i)\right|+\sum_{j=1, j \neq i}^{n} \frac{q_{j}(i)}{q_{i}(i)}\left|w_{j i}(i)\right| L_{i}^{2}+\sum_{j=1}^{n}\left|h_{i j}(i)\right| L_{j} \\
& +\sum_{j=1}^{n}\left|b_{i j}(i)\right|+\sum_{j=1}^{n} \frac{q_{j}(i)}{q_{i}(i)} L_{i}^{2}\left|b_{j i}(i)\right| \int_{0}^{+\infty} k_{j i}(s) e^{2 \alpha_{i} s} d s+\sum_{j=1}^{n} \frac{\left|h_{j i}(i)\right|}{1-\mu} e^{2 \alpha \tau} \frac{q_{j}}{q_{i}} L_{i} \\
& +\sum_{j=1}^{n} \gamma_{i j} q_{i}(j)+1 \\
= & 0 .
\end{aligned}
$$

Let $\alpha=\min _{1 \leq i \leq n}\left\{\alpha_{i}\right\}$. Clearly, $\alpha>0$, and we can get

$$
\begin{aligned}
F_{i}(\alpha)= & 2 \alpha-\Xi-2 a_{i}(i)+2\left|w_{i i}(i)\right| L_{i}+\sum_{j=1, j \neq i}^{n}\left|w_{i j}(i)\right|+\sum_{j=1, j \neq i}^{n} \frac{q_{j}(i)}{q_{i}(i)}\left|w_{j i}(i)\right| L_{i}^{2}+\sum_{j=1}^{n}\left|h_{i j}(i)\right| L_{j} \\
& +\sum_{j=1}^{n}\left|b_{i j}(i)\right|+\sum_{j=1}^{n} \frac{q_{j}(i)}{q_{i}(i)}\left|b_{j i}(i)\right| L_{i}^{2} \int_{0}^{+\infty} k_{j i}(s) e^{2 \alpha s} d s+\sum_{j=1}^{n} \frac{\left|h_{j i}(i)\right|}{1-\mu} e^{2 \alpha \tau} \frac{q_{j}}{q_{i}} L_{i} \\
& +\sum_{j=1}^{n} \gamma_{i j} q_{i}(j)+1 \\
\leq & 0 .
\end{aligned}
$$


Given $\varphi \in L_{F_{0}}^{p}\left((-\infty, 0] \times \Omega ; R^{n}\right)$, fix the system mode $i \in S$ arbitrarily. Let $\phi_{j}(t)=t-\tau_{j}(t)$. Since the derivative $\dot{\phi}_{j}(t)=1-\dot{\tau}_{j}(t) \geq 1-\mu>0, \phi_{j}(t)$ has an inverse function. We denote this inverse function by $\phi_{j}^{-1}(t)$. Construct the Lyapunov functional

$$
\begin{aligned}
V(t, u(t), r(t)=i)= & \int_{\Omega} \sum_{i=1}^{n} q_{i}(i)\left[e^{2 \alpha t} u_{i}(t)^{2}\right. \\
& +\frac{1}{1-\mu} \sum_{j=1}^{n}\left|h_{i j}(i)\right| L_{j} \int_{t-\tau_{j}(t)}^{t} u_{j}(s)^{2} e^{2 \alpha\left(s+\tau_{j}\left(\phi_{j}^{-1}(s)\right)\right)} d s \\
& \left.+\sum_{j=1}^{n}\left|b_{i j}(i)\right| \int_{0}^{+\infty} k_{i j}(s) \int_{t-s}^{t} g_{j}\left(u_{j}(z)\right)^{2} e^{2 \alpha(z+s)} d z d s\right] d x .
\end{aligned}
$$

Along the solutions of model (1), we have

$$
\begin{aligned}
& L V(t, u(t), r(t)=i)=\lim _{\Delta \rightarrow 0^{+}} \frac{1}{\Delta}[E\{V(t+\Delta, u(t+\Delta), r(t+\Delta)) \mid u(t), r(t)=i\} \\
& -V(t, u(t), r(t)=i)] \\
& =\int_{\Omega} e^{2 \alpha t} \sum_{i=1}^{n} q_{i}(i)\left\{2 u _ { i } ( t ) \left[\sum_{l=1}^{m} \frac{\partial}{\partial x_{l}}\left(D_{i l} \frac{\partial u_{i}(t)}{\partial x_{l}}\right)-a_{i}(i) u_{i}(t)\right.\right. \\
& +\sum_{j=1}^{n} w_{i j}(i) g_{j}\left(u_{j}(t)\right)+\sum_{j=1}^{n} h_{i j}(i) g_{j}\left(u_{j}\left(t-\tau_{j}(t)\right)\right) \\
& \left.+\sum_{j=1}^{n} b_{i j}(i) \int_{-\infty}^{t} k_{i j}(t-s) g_{j}\left(u_{j}(s)\right) d s+v_{i}(t)\right]+2 \alpha u_{i}^{2}(t) \\
& +\sum_{j=1}^{n} \frac{\left|h_{i j}(i)\right|}{1-\mu} L_{j}\left[e^{2 \alpha \tau} u_{j}(t)^{2}-(1-\mu) u_{j}\left(t-\tau_{j}(t)\right)^{2}\right] \\
& +e^{2 \alpha t} \sum_{j=1}^{n} \gamma_{i j} q_{i}(j) u_{i}(t)^{2} \\
& +\sum_{j=1}^{n}\left|b_{i j}(i)\right|\left[\int_{0}^{+\infty} e^{2 \alpha s} k_{i j}(s) g_{j}\left(u_{j}(t)\right)^{2} d s\right. \\
& \left.\left.-\int_{0}^{+\infty} k_{i j}(s) g_{j}\left(u_{j}(t-s)\right)^{2} d s\right]\right\} d x \\
& \leq \int_{\Omega} e^{2 \alpha t} \sum_{i=1}^{n} q_{i}(i)\left\{\left[2 u_{i}(t) \sum_{l=1}^{m} \frac{\partial}{\partial x_{l}}\left(D_{i l} \frac{\partial u_{i}(t)}{\partial x_{l}}\right)-2 a_{i}(i) u_{i}(t)^{2}\right.\right. \\
& +2\left|w_{i i}(i)\right| L_{i} u_{i}(t)^{2}+2 \sum_{j=1, j \neq i}^{n}\left|w_{i j}(i)\right|\left|u_{i}(t)\right|\left|g_{j}\left(u_{j}(t)\right)\right| \\
& +2\left|u_{i}(t)\right| \sum_{j=1}^{n}\left|h_{i j}(i)\right| L_{j}\left|u_{j}\left(t-\tau_{j}(t)\right)\right| \\
& \left.+2\left|u_{i}(t)\right| \sum_{j=1}^{n}\left|b_{i j}(i)\right| \int_{-\infty}^{t} k_{i j}(t-s)\left|g_{j}\left(u_{j}(s)\right)\right| d s+2\left|u_{i}(t)\right| v_{i}(t)\right]
\end{aligned}
$$




$$
\begin{aligned}
& +2 \alpha u_{i}(t)^{2}+\sum_{j=1}^{n} \frac{\left|h_{i j}(i)\right|}{1-\mu} L_{j}\left[e^{2 \alpha \tau} u_{j}(t)^{2}-(1-\mu) u_{j}\left(t-\tau_{j}(t)\right)^{2}\right] \\
& +\sum_{j=1}^{n} \gamma_{i j} q_{i}(j) u_{i}(t)^{2} \\
& +\sum_{j=1}^{n}\left|b_{i j}(i)\right|\left[\int_{0}^{+\infty} k_{i j}(s) e^{2 \alpha s}\left|g_{j}\left(u_{j}(t)\right)\right|^{2} d s\right. \\
& \left.\left.-\int_{0}^{+\infty} k_{i j}(s)\left|g_{j}\left(u_{j}(t-s)\right)\right|^{2} d s\right]\right\} d x .
\end{aligned}
$$

From Young's inequality and (A2), we obtain

$$
2 \sum_{j=1, j \neq i}^{n}\left|w_{i j}(i)\right|\left|u_{i}(t)\right|\left|g_{j}\left(u_{j}(t)\right)\right| \leq \sum_{j=1, j \neq i}^{n}\left|w_{i j}(i)\right|\left|u_{i}(t)\right|^{2}+\sum_{j=1, j \neq i}^{n}\left|w_{i j}(i)\right|\left|g_{j}\left(u_{j}(t)\right)\right|^{2}
$$

and

$$
\begin{aligned}
& 2\left|u_{i}(t)\right| \sum_{j=1}^{n}\left|b_{i j}(i)\right| \int_{-\infty}^{t} k_{i j}(t-s)\left|g_{j}\left(u_{j}(s, x)\right)\right| d s \\
& \leq \sum_{j=1}^{n}\left|b_{i j}(i)\right|\left|u_{i}(t)\right|^{2}+\sum_{j=1}^{n}\left|b_{i j}(i)\right| \int_{-\infty}^{t} k_{i j}(t-s)\left|g_{j}\left(u_{j}(s, x)\right)\right|^{2} d s .
\end{aligned}
$$

Applying the Green formula, the Dirichlet boundary condition, and Lemma 1, we have

$$
\begin{aligned}
2 \int_{\Omega} \sum_{l=1}^{m} u_{i}(t) \frac{\partial}{\partial x_{l}}\left(D_{i l} \frac{\partial u_{i}(t)}{\partial x_{l}}\right) d x & =-2 \sum_{l=1}^{m} \int_{\Omega} D_{i l}\left(\frac{\partial u_{i}(t)}{\partial x_{l}}\right)^{2} d x \\
& <-\left(\frac{\underline{\alpha}(m-2)^{2}}{2 \pi^{2}}+\frac{2 \underline{\alpha} \Lambda_{2}}{R_{\Omega}^{2}}\right) \int_{\Omega} u_{i}(t)^{2} d x \\
& =-\Xi \int_{\Omega} u_{i}(t)^{2} d x .
\end{aligned}
$$

By (11) -(13) and (A2) we derive

$$
\begin{aligned}
L V(t, u, i) \leq & \int_{\Omega} e^{2 \alpha t} \sum_{i=1}^{n} q_{i}(i)\left\{\left[-\Xi u_{i}(t)^{2}-2 a_{i}(i) u_{i}(t)^{2}\right.\right. \\
& +2\left|w_{i i}(i)\right| L_{i} u_{i}(t)^{2}+\sum_{j=1, j \neq i}^{n}\left|w_{i j}(i)\right|\left|u_{i}(t)\right|^{2}+\sum_{j=1, j \neq i}^{n}\left|w_{i j}(i)\right| L_{j}^{2}\left|u_{j}(t)\right|^{2} \\
& +\sum_{j=1}^{n}\left|h_{i j}(i)\right| L_{j}\left(\left|u_{i}(t)\right|^{2}+\left|u_{j}\left(t-\tau_{j}(t)\right)\right|^{2}\right)+\sum_{j=1}^{n}\left|b_{i j}(i)\right|\left|u_{i}(t)\right|^{2} \\
& \left.+\sum_{j=1}^{n}\left|b_{i j}(i)\right| \int_{-\infty}^{t} k_{i j}(t-s)\left|g_{j}\left(u_{j}(s)\right)\right|^{2} d s+\left|u_{i}(t)\right|^{2}+v_{i}(t)^{2}\right] \\
& +\sum_{j=1}^{n} \frac{\left|h_{i j}(i)\right|}{1-\mu} L_{j} e^{2 \alpha \tau}\left|u_{j}(t)\right|^{2}
\end{aligned}
$$




$$
\begin{aligned}
& -\sum_{j=1}^{n}\left|h_{i j}(i)\right| L_{j}\left|u_{j}\left(t-\tau_{j}(t)\right)\right|^{2}+\sum_{j=1}^{n} \gamma_{i j} q_{i}(j) u_{i}(t)^{2}+2 \alpha u_{i}(t)^{2} \\
& +\sum_{j=1}^{n}\left|b_{i j}(i)\right|\left[\int_{0}^{+\infty} k_{i j}(s) e^{2 \alpha s}\left|g_{j}\left(u_{j}(t)\right)\right|^{2} d s\right. \\
& \left.\left.-\int_{0}^{+\infty} k_{i j}(s)\left|g_{j}\left(u_{j}(t-s)\right)\right|^{2} d s\right]\right\} d x \\
& =\int_{\Omega} e^{2 \alpha t} \sum_{i=1}^{n} q_{i}(i)\left[\left(-\Xi-2 a_{i}(i)+2\left|w_{i i}(i)\right| L_{i}+\sum_{j=1, j \neq i}^{n}\left|w_{i j}(i)\right|\right.\right. \\
& +\sum_{j=1, j \neq i}^{n} \frac{q_{j}(i)}{q_{i}(i)}\left|w_{j i}(i)\right| L_{i}^{2} \\
& +\sum_{j=1}^{n}\left|h_{i j}(i)\right| L_{j}+\sum_{j=1}^{n}\left|b_{i j}(i)\right|+\sum_{j=1}^{n} \frac{q_{j}(i)}{q_{i}(i)}\left|b_{j i}(i)\right| L_{i}^{2} \int_{0}^{+\infty} k_{j i}(s) e^{2 \alpha s} d s \\
& \left.\left.+\sum_{j=1}^{n} \frac{\left|h_{j i}(i)\right|}{1-\mu} e^{2 \alpha \tau} \frac{q_{j}(i)}{q_{i}(i)} L_{i}+1+2 \alpha+\sum_{j=1}^{n} \gamma_{i j} q_{i}(j)\right)\left|u_{i}(t)\right|^{2}+v_{i}(t)^{2}\right] d x .
\end{aligned}
$$

It follows from Dynkin's formula and (14) that

$$
\begin{aligned}
E V(t, u, i) \leq & E V(0, \varphi(0), i)+\left\{\int _ { 0 } ^ { t } e ^ { 2 \alpha \xi } \sum _ { i = 1 } ^ { n } q _ { i } ( i ) \left[\left(-\Xi-2 a_{i}(i)+2\left|w_{i i}(i)\right| L_{i}\right.\right.\right. \\
& +\sum_{j=1, j \neq i}^{n}\left|w_{i j}(i)\right|+\sum_{j=1, j \neq i}^{n} \frac{q_{j}(i)}{q_{i}(i)}\left|w_{j i}(i)\right| L_{i}^{2}+\sum_{j=1}^{n}\left|h_{i j}(i)\right| L_{j}+\sum_{j=1}^{n}\left|b_{i j}(i)\right| \\
& +\sum_{j=1}^{n} \frac{q_{j}(i)}{q_{i}(i)}\left|b_{j i}(i)\right| L_{i}^{2} \int_{0}^{+\infty} k_{j i}(s) e^{2 \alpha s} d s \\
& \left.\left.\left.+\sum_{j=1}^{n} \frac{\left|h_{j i}(i)\right|}{1-\mu} e^{2 \alpha \tau} \frac{q_{j}(i)}{q_{i}(i)} L_{i}+1+2 \alpha+\sum_{j=1}^{n} \gamma_{i j} q_{i}(j)\right) E\left\|u_{i}(\xi)\right\|_{2}^{2}\right] d \xi\right\} \\
& +\frac{n}{2 \alpha} E\|v(t)\|_{\Omega}^{2}\left(e^{2 \alpha t}-1\right) .
\end{aligned}
$$

Since

$$
V(t, u, i) \geq \sum_{i=1}^{n} q_{i}(i) e^{2 \alpha t}\left\|u_{i}(t)\right\|_{2}^{2} \geq \min _{1 \leq i \leq n}\left\{q_{i}(i)\right\} e^{2 \alpha t} \sum_{i=1}^{n}\left\|u_{i}(t)\right\|_{2}^{2}, \quad t \geq 0
$$

and

$$
\begin{aligned}
V(0, \varphi(0), 0)= & \int_{\Omega} \sum_{i=1}^{n} q_{i}(i)\left[\varphi_{i}(0)^{2}+\frac{1}{1-\mu} \sum_{j=1}^{n}\left|h_{i j}(0)\right| L_{j} \int_{-\tau_{j}(0)}^{0} u_{j}^{2}(s, x) e^{2 \alpha\left(s+\tau_{j}\left(\psi_{j}^{-1}(s)\right)\right)} d s\right. \\
& \left.+\sum_{j=1}^{n}\left|b_{i j}(0)\right| \int_{0}^{+\infty} k_{i j}(s) \int_{-s}^{0} g_{j}\left(u_{j}(z, x)\right)^{2} e^{2 \alpha(z+s)} d z d s\right] d x
\end{aligned}
$$




$$
\begin{aligned}
\leq & \max _{1 \leq i \leq n}\left\{q_{i}(0)\right\} \sum_{i=1}^{n}\left\{\left\|\varphi_{i}(0)\right\|_{2}^{2}\right. \\
& +\sum_{j=1}^{n}\left|b_{i j}(0)\right| L_{j}^{2} \int_{0}^{+\infty} k_{i j}(s)\left[\int_{-s}^{0}\left\|u_{j}(z, x)\right\|_{2}^{2} e^{2 \alpha(z+s)} d z\right] d s \\
& \left.+\frac{1}{1-\mu} \sum_{j=1}^{n}\left|h_{i j}(0)\right| L_{j} \int_{-\tau}^{0}\left\|u_{i}(s)\right\|_{2}^{2} e^{2 \alpha\left(s+\tau_{j}\left(\psi_{j}^{-1}(s)\right)\right)} d s\right\} \\
\leq & \max _{1 \leq i \leq n}\left\{q_{i}(0)\right\}\left\{1+\max _{1 \leq i \leq n}\left\{\sum_{j=1}^{n}\left|b_{j i}(0)\right| L_{i}^{2} \int_{0}^{+\infty} s e^{2 \alpha s} k_{j i}(s) d s\right\}\right. \\
& \left.+\frac{\tau e^{2 \alpha \tau}}{1-\mu} \sum_{j=1}^{n}\left|h_{i j}(0)\right| L_{j}\right\}\left\|\varphi_{i}\right\|_{2}^{2},
\end{aligned}
$$

combining (4) and (15)-(17), we derive

$$
\begin{aligned}
E\left[\|u(t)\|_{2}\right] \leq & \left(\frac{\max _{1 \leq i \leq n}\left\{q_{i}(0)\right\}}{\min _{1 \leq i \leq n}\left\{q_{i}(i)\right\}}\right)^{1 / 2} e^{-\alpha t}\left\{1+\max _{1 \leq i \leq n}\left\{\sum_{j=1}^{n}\left|b_{j i}(0)\right| L_{i}^{2} \int_{0}^{+\infty} s e^{2 \alpha s} k_{j i}(s) d s\right\}\right. \\
& \left.+\frac{\tau e^{2 \alpha \tau}}{1-\mu} \sum_{j=1}^{n}\left|h_{i j}(0)\right| L_{j}\right\}^{1 / 2} E[\|\varphi\|]+\left(\frac{n}{2 \alpha \min _{1 \leq i \leq n}\left\{q_{i}(i)\right\}}\right)^{1 / 2} E\left[\|v(t)\|_{\Omega}\right] .
\end{aligned}
$$

Hence, from (3) we get that system (1)-(2) is almost sure ISS. This completes the proof of Theorem 1.

Remark 4 In this paper, we concern with the Markovian jump RDNNs with Dirichlet boundary conditions. The results are expressed by a set of inequalities. These conditions are easy to verify, and our results play an important role in the design and applications of almost sure ISS. It is worth mentioning that the effect of reaction-diffusion terms is considered by the Hardy-Poincaré inequality. In Theorem 1, the Hardy-Poincaré inequality is used firstly. Moreover, we can see a very interesting fact that as long as diffusion coefficients $D_{i l}$ in system (1) are large enough, (4) always be satisfied. This shows that a large enough diffusion can always make system (1)-(2) almost sure ISS.

Remark 5 If we do not consider Markov jump parameters, that is, the Markov chain $\{r(t), t \geq 0\}$ only takes a unique value 1 (i.e., $S=\{1\}$ ), then, for simplicity, we write $a_{i}(1)=a_{i}$, $w_{i j}(1)=w_{i j}, h_{i j}(1)=h_{i j}, b_{i j}(1)=b_{i j}$. Then system (1) will be reduced to the following deterministic delayed RDNNs:

$$
\begin{aligned}
\frac{\partial u_{i}(t, x)}{\partial t}= & \sum_{l=1}^{m} \frac{\partial}{\partial x_{l}}\left(D_{i l} \frac{\partial u_{i}(t, x)}{\partial x_{l}}\right)-a_{i} u_{i}(t, x)+\sum_{j=1}^{n} w_{i j} g_{j}\left(u_{j}(t, x)\right) \\
& +\sum_{j=1}^{n} h_{i j} g_{j}\left(u_{j}\left(t-\tau_{j}(t), x\right)\right)+\sum_{j=1}^{n} b_{i j} \int_{-\infty}^{t} k_{i j}(t-s) g_{j}\left(u_{j}(s, x)\right) d s \\
& +v_{i}(t), \quad t \geq 0, x \in \Omega .
\end{aligned}
$$

It is worth pointing out that particular cases of system (19) were studied in [19, 23]. 
The next theorem shows that the equilibrium solution of system (19) is ISS. The proof of Theorem 2 is similar to that in Theorem 1, and thus we omit it.

Theorem 2 Suppose that (A1)-(A2) hold. System (19) and (2) is ISS if there exist constants $q_{i}>0$ for any $i, j=1,2, \ldots, n$ such that

$$
\begin{aligned}
-\Xi & -2 a_{i}+2\left|w_{i i}\right| L_{i}+\sum_{j=1, j \neq i}^{n}\left|w_{i j}\right|+\sum_{j=1, j \neq i}^{n} \frac{q_{j}}{q_{i}}\left|w_{j i}\right| L_{i}^{2}+\sum_{j=1}^{n}\left|h_{i j}\right| L_{j}+\sum_{j=1}^{n}\left|b_{i j}\right| \\
& +\sum_{j=1}^{n} \frac{q_{j}}{q_{i}}\left|b_{j i}\right| L_{i}^{2}+\sum_{j=1}^{n} \frac{\left|h_{j i}\right|}{1-\mu} e^{2 \alpha \tau} \frac{q_{j}}{q_{i}} L_{i}+1<0 .
\end{aligned}
$$

Remark 6 Theorem 1 reduces to almost sure exponential stability condition for delayed RDNNs with Markovian jump parameters if $v(t)=0$. Similarly, Theorem 2 becomes an exponential stability condition for delayed RDNNs when $v(t)=0$. In [34], the authors employed the Lyapunov direct method to consider the almost sure stability of Itô stochastic reaction-diffusion systems with Brownian motion defined in a complete probability space, including asymptotic stability in probability and almost sure exponential stability. In addition, the stability criteria in [34] are independent on reaction-diffusion coefficients and the regional feature. Compared with [34], this paper studies the ISS analysis for a class of RDNNs with mixed delays and Markovian jump parameters. Furthermore, the given ISS criteria are true to Dirichlet boundary conditions and concerned with the regional feature, the reaction-diffusion coefficients, and the first eigenvalue of the Dirichlet Laplacian.

Some famous NN models are particular cases of model (1). In system (1)-(2), ignoring the role of reaction-diffusion, system (1) reduces to the following delayed NNs:

$$
\begin{aligned}
& d u_{i}(t)= {\left[-a_{i}(r(t)) u_{i}(t)+\sum_{j=1}^{n} w_{i j}(r(t)) g_{j}\left(u_{j}(t)\right)+\sum_{j=1}^{n} h_{i j}(r(t)) g_{j}\left(u_{j}\left(t-\tau_{j}(t)\right)\right)\right.} \\
&\left.+\sum_{j=1}^{n} b_{i j}(r(t)) \int_{-\infty}^{t} k_{i j}(t-s) g_{j}\left(u_{j}(s)\right) d s+v_{i}(t)\right] d t, \quad t \geq 0, \\
& u_{i}(s)=\varphi_{i}(s), \quad s \in(-\infty, 0] .
\end{aligned}
$$

As a consequence of Theorems 1 and 2, we get the following results.

Corollary 1 Assume that (A1) and (A2) are satisfied. System (21) is almost sure ISS if there exist constants $q_{i}(i)>0$ for any $r(t)=i \in S, i, j=1,2, \ldots, n$, such that

$$
\begin{aligned}
-2 a_{i}(i) & +2\left|w_{i i}(i)\right| L_{i}+\sum_{j=1, j \neq i}^{n}\left|w_{i j}(i)\right|+\sum_{j=1, j \neq i}^{n} \frac{q_{j}(i)}{q_{i}(i)}\left|w_{j i}(i)\right| L_{i}^{2}+\sum_{j=1}^{n}\left|h_{i j}(i)\right| L_{j}+\sum_{j=1}^{n}\left|b_{i j}(i)\right| \\
+ & \sum_{j=1}^{n} \frac{q_{j}(i)}{q_{i}(i)}\left|b_{j i}(i)\right| L_{i}^{2}+\sum_{j=1}^{n} \frac{\left|h_{j i}(i)\right|}{1-\mu} e^{2 \alpha \tau} \frac{q_{j}(i)}{q_{i}(i)} L_{i}+\sum_{j=1}^{n} \gamma_{i j} q_{i}(j)+1
\end{aligned}
$$

$<0$. 
Corollary 2 Assume that (A1) and (A2) are satisfied. System (21) is ISS if there exist constants $q_{i}>0$ for any $i, j=1,2, \ldots, n$ such that

$$
\begin{aligned}
& -2 a_{i}+2\left|w_{i i}\right| L_{i}+\sum_{j=1, j \neq i}^{n}\left|w_{i j}\right|+\sum_{j=1, j \neq i}^{n} \frac{q_{j}}{q_{i}}\left|w_{j i}\right| L_{i}^{2}+\sum_{j=1}^{n}\left|h_{i j}\right| L_{j}+\sum_{j=1}^{n}\left|b_{i j}\right| \\
& +\sum_{j=1}^{n} \frac{q_{j}}{q_{i}}\left|b_{j i}\right| L_{i}^{2}+\sum_{j=1}^{n} \frac{\left|h_{j i}\right|}{1-\mu} e^{2 \alpha \tau} \frac{q_{j}}{q_{i}} L_{i}+1<0 .
\end{aligned}
$$

Remark 7 Our model in (21) is more general than some well-studied NNs. When $b_{i j}=0$, the model in (21) reduces the model studied in [15]. The authors in [15] present criteria for the ISS of NNs with time-varying delays. Corollary 2 in this paper is much less conservative than those in [15]. Moreover, our results depend on Markovian jump parameters and can be easily checked by simple computation. To the best of authors' knowledge, up to now, little work is reported on almost sure ISS of NNs with Markovian jump parameters and mixed time-varying delays.

\section{Illustrative examples}

We present two examples to illustrate the usefulness of our main results. Our aim is to examine the almost sure ISS of given RDNNs with Markovian jump parameters and mixed time delays.

Example 1 Consider the two-neuron delayed RDNNs with Markovian jump parameters

$$
\begin{aligned}
& \frac{\partial u_{i}(t, x)}{\partial t}= \sum_{l=1}^{m} \frac{\partial}{\partial x_{l}}\left(D_{i l} \frac{\partial u_{i}(t, x)}{\partial x_{l}}\right)-a_{i}(r(t)) u_{i}(t, x)+\sum_{j=1}^{n} w_{i j}(r(t)) g_{j}\left(u_{j}(t, x)\right) \\
&+\sum_{j=1}^{n} h_{i j}(r(t)) g_{j}\left(u_{j}\left(t-\tau_{j}(t), x\right)\right)+\sum_{j=1}^{n} b_{i j}(r(t)) \int_{-\infty}^{t} k_{i j}(t-s) g_{j}\left(u_{j}(s, x)\right) d s \\
&+v_{i}(t), \quad t \geq 0, x \in \Omega, \\
& u_{i}(t, x)=0, \quad(t, x) \in[0,+\infty) \times \partial \Omega, \quad u_{i}(s, x)=\varphi_{i}(s, x), \quad(s, x) \in(-\infty, 0] \times \Omega, \quad(24)
\end{aligned}
$$

where $\Omega=\left\{\left(x_{1}, \ldots, x_{4}\right)^{T} \mid-1<x_{k}<1, k=1, \ldots, 4\right\} \subset R^{4}, k_{i j}(s)=s e^{-s}, n=2, m=3, \pi=2$, $R_{\Omega}=2, \mu=0.5, \Lambda_{2}=5.783, \tau=\ln 2, \alpha=0.5, g_{j}(\eta)=\frac{1}{2}(|\eta+1|-|\eta-1|), L_{j}=1, D_{i l}=1$, $i, j=1,2, l=1, \ldots, 4, \tau_{1}(t)=\tau_{2}(t)=0.5(1+\sin t), v(t)=[\sin t \cos 2 t]^{T}$, and the generator of the Markov chain and parameters are

$$
\begin{aligned}
& \Gamma=\left(\begin{array}{cc}
-0.1 & 0.1 \\
0.2 & -0.2
\end{array}\right), \quad A(1)=\left(\begin{array}{ll}
3 & 0 \\
0 & 2
\end{array}\right), \quad A(2)=\left(\begin{array}{cc}
3 & 0 \\
0 & 1.5
\end{array}\right) \\
& W(1)=\left(\begin{array}{cc}
0.2 & -0.1 \\
0.1 & -0.3
\end{array}\right), \quad W(2)=\left(\begin{array}{cc}
0.1 & 0 \\
0.1 & -0.1
\end{array}\right), \\
& H(1)=\left(\begin{array}{cc}
0.2 & 0 \\
0 & 0.1
\end{array}\right), \quad H(2)=\left(\begin{array}{cc}
0.5 & -0.1 \\
0.3 & 0.1
\end{array}\right),
\end{aligned}
$$




$$
B(1)=\left(\begin{array}{cc}
0 & 1 \\
1 & -1
\end{array}\right), \quad B(2)=\left(\begin{array}{cc}
1 & 0 \\
-1 & 1
\end{array}\right) .
$$

Then a simple computation yields

$$
\begin{aligned}
-\Xi- & 2 a_{1}(1)+2\left|w_{11}(1)\right| L_{1} \\
& +\sum_{j=1, j \neq i}^{n}\left|w_{1 j}(1)\right|+\sum_{j=1, j \neq i}^{n} \frac{q_{j}(1)}{q_{1}(1)}\left|w_{j 1}(1)\right| L_{1}^{2}+\sum_{j=1}^{n}\left|h_{1 j}(1)\right| L_{j}+\sum_{j=1}^{n}\left|b_{1 j}(1)\right| \\
& +\sum_{j=1}^{n} \frac{q_{j}(1)}{q_{1}(1)}\left|b_{j 1}(1)\right| L_{1}^{2}+\sum_{j=1}^{n} \frac{\left|h_{j 1}(1)\right|}{1-\mu} e^{2 \alpha \tau} \frac{q_{j}(1)}{q_{1}(1)} L_{1}+\sum_{j=1}^{n} \gamma_{1 j} q_{1}(j)+1 \\
= & -6.21<0, \\
-\Xi- & 2 a_{2}(1)+2\left|w_{22}(1)\right| L_{2} \\
& +\sum_{j=1, j \neq i}^{n}\left|w_{2 j}(1)\right|+\sum_{j=1, j \neq i}^{n} \frac{q_{j}(1)}{q_{2}(1)}\left|w_{j 2}(1)\right| L_{2}^{2}+\sum_{j=1}^{n}\left|h_{2 j}(1)\right| L_{j}+\sum_{j=1}^{n}\left|b_{2 j}(1)\right| \\
& +\sum_{j=1}^{n} \frac{q_{j}(1)}{q_{2}(1)}\left|b_{j 2}(1)\right| L_{2}^{2}+\sum_{j=1}^{n} \frac{\left|h_{j 2}(1)\right|}{1-\mu} e^{2 \alpha \tau} \frac{q_{j}(1)}{q_{2}(1)} L_{2}+\sum_{j=1}^{n} \gamma_{2 j} q_{2}(j)+1 \\
= & -3.61<0 . \\
-\Xi- & 2 a_{1}(2)+2\left|w_{11}(2)\right| L_{1} \\
& +\sum_{j=1, j \neq i}^{n}\left|w_{1 j}(2)\right|+\sum_{j=1, j \neq i}^{n} \frac{q_{j}(2)}{q_{1}(2)}\left|w_{j 1}(2)\right| L_{1}^{2}+\sum_{j=1}^{n}\left|h_{1 j}(2)\right| L_{j}+\sum_{j=1}^{n}\left|b_{1 j}(2)\right| \\
& +\sum_{j=1}^{n} \frac{q_{j}(2)}{q_{2}(2)}\left|b_{j 2}(2)\right| L_{2}^{2}+\sum_{j=1}^{n} \frac{\left|h_{j 2}(2)\right|}{1-\mu} e^{2 \alpha \tau} \frac{q_{j}(2)}{q_{2}(2)} L_{2}+\sum_{j=1}^{n} \gamma_{2 j} q_{2}(j)+1 \\
& +\sum_{j=1}^{n} \frac{q_{j}(2)}{q_{1}(2)}\left|b_{j 1}(2)\right| L_{1}^{2}+\sum_{j=1}^{n} \frac{\left|h_{j 1}(2)\right|}{1-\mu} e^{2 \alpha \tau} \frac{q_{j}(2)}{q_{1}(2)} L_{1}+\sum_{j=1}^{n} \gamma_{1 j} q_{1}(j)+1 \\
= & -4.31<0, \\
-\Xi- & 2 a_{2}(2)+2\left|w_{22}(2)\right| L_{2} \\
& +\sum_{j=1, j \neq i}^{n} \frac{q_{j}(2)}{q_{2}(2)}\left|w_{j 2}(2)\right| L_{2}^{2}+\sum_{j=1}^{n}\left|h_{2 j}(2)\right| L_{j}+\sum_{j=1}^{n}\left|b_{2 j}(2)\right| \\
&
\end{aligned}
$$

According to Theorem 1, system (24) is almost sure ISS. Figures 1-6 show that the state of RDNNs (24) in the presence of input and illustrate the feature of ISS that its behavior remains bounded when its inputs are bounded for RDNNs (24). The evolutions of states are shown in Figs. 1-6, which also demonstrate that system (24) is almost sure ISS, whereas Fig. 7 is given to show the situation of the Markovian switching sequence. 


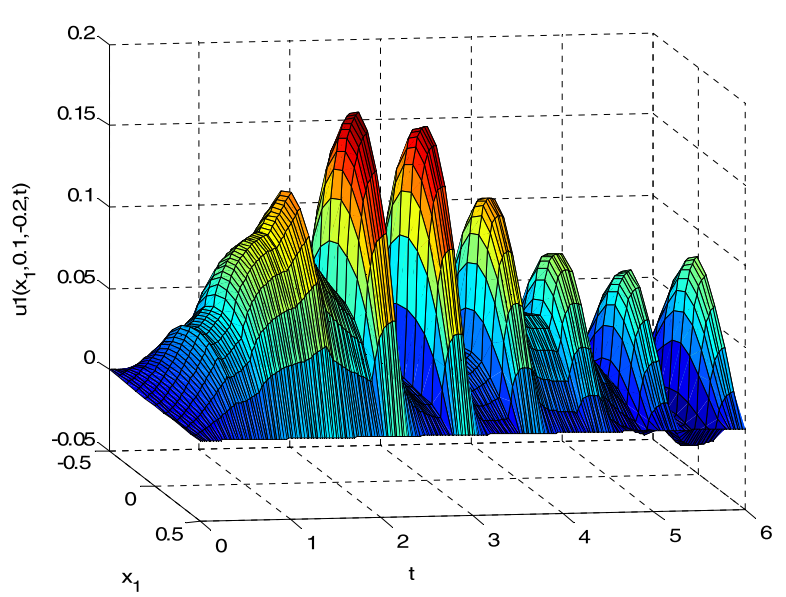

Figure 1 The state surface of $u_{1}\left(x_{1}, 0.1,-0.2, t\right)$

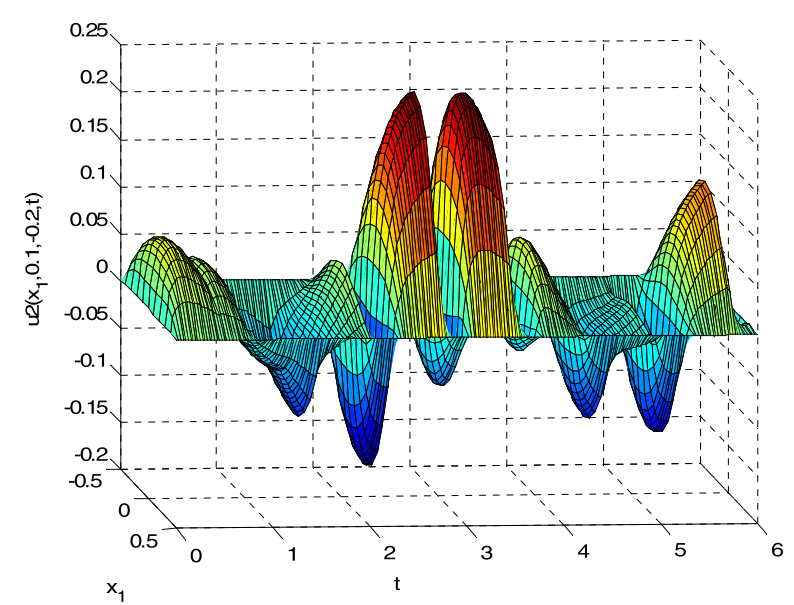

Figure 2 The state surface of $u_{2}\left(x_{1}, 0.1,-0.2, t\right)$

Example 2 Consider the two-neuron delayed RDNNs with Markovian jump parameters

$$
\begin{aligned}
& \frac{\partial u_{i}(t, x)}{\partial t}= \sum_{l=1}^{m} \frac{\partial}{\partial x_{l}}\left(D_{i l} \frac{\partial u_{i}(t, x)}{\partial x_{l}}\right)-a_{i}(r(t)) u_{i}(t, x)+\sum_{j=1}^{n} w_{i j}(r(t)) g_{j}\left(u_{j}(t, x)\right) \\
&+\sum_{j=1}^{n} h_{i j}(r(t)) g_{j}\left(u_{j}\left(t-\tau_{j}(t), x\right)\right)+\sum_{j=1}^{n} b_{i j}(r(t)) \int_{-\infty}^{t} k_{i j}(t-s) g_{j}\left(u_{j}(s, x)\right) d s \\
&+v_{i}(t), \quad t \geq 0, x \in \Omega, \\
& u_{i}(t, x)=0, \quad(t, x) \in[0,+\infty) \times \partial \Omega, \quad u_{i}(s, x)=\varphi_{i}(s, x), \quad(s, x) \in(-\infty, 0] \times \Omega,
\end{aligned}
$$

where $\Omega=\left\{\left(x_{1}, \ldots, x_{4}\right)^{T} \mid-1<x_{k}<1, k=1, \ldots, 4\right\} \subset R^{4}, k_{i j}(s)=s e^{-s}, n=2, m=3, \pi=2$, $R_{\Omega}=2, \mu=0.5, \Lambda_{2}=5.783, \tau=\ln 2, \alpha=0.5, g_{j}(\eta)=\tanh (\eta), x=\left(x_{1}, 0.1,-0.2\right) L_{j}=1, D_{i l}=1$, $i, j=1,2, l=1, \ldots, 4, \tau_{1}(t)=\tau_{2}(t)=0.5(1+\sin t), v(t)=[\sin 2 t \cos t]^{T}$, and the generator of 


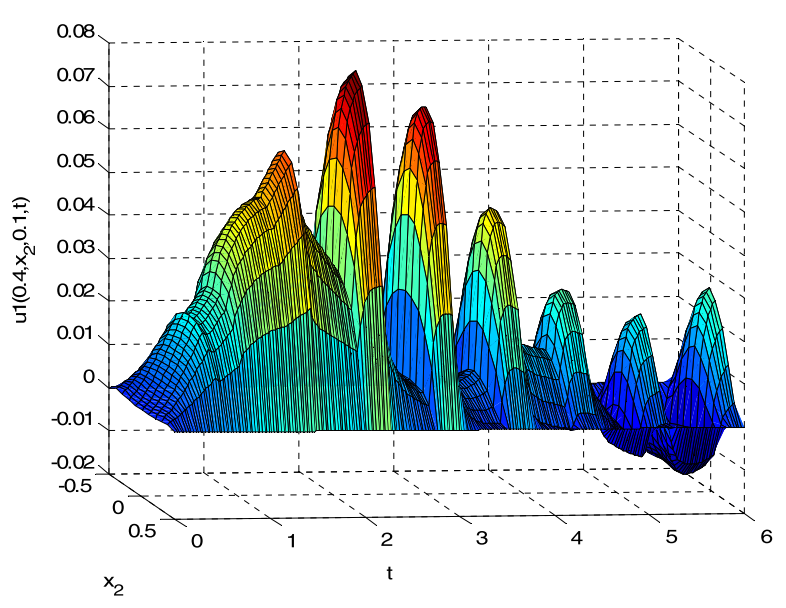

Figure 3 The state surface of $u_{1}\left(0.4, x_{2}, 0.1, t\right)$

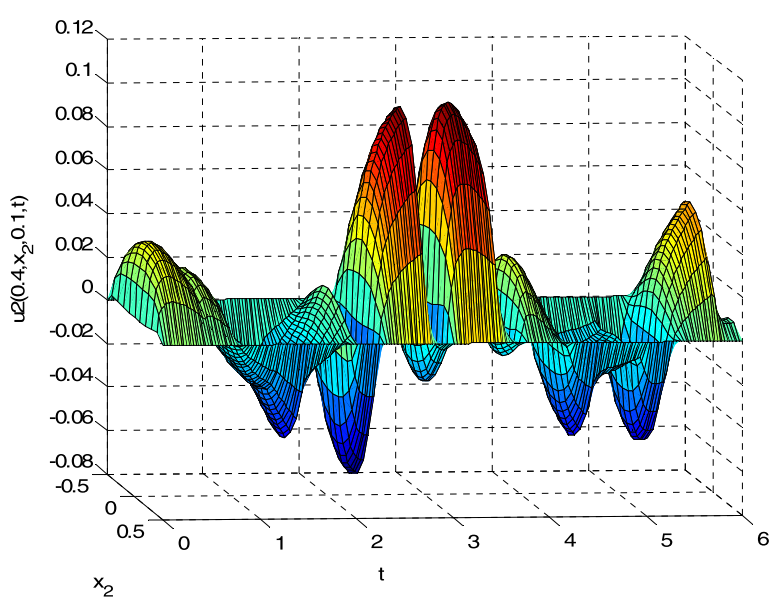

Figure 4 The state surface of $u_{2}\left(0.4, x_{2}, 0.1, t\right)$

the Markov chain and parameters are

$$
\begin{aligned}
& \Gamma=\left(\begin{array}{cc}
-0.1 & 0.1 \\
0.2 & -0.2
\end{array}\right), \quad A(1)=\left(\begin{array}{cc}
2 & 0 \\
0 & 1.5
\end{array}\right), \quad A(2)=\left(\begin{array}{cc}
2 & 0 \\
0 & 1.8
\end{array}\right), \\
& W(1)=\left(\begin{array}{cc}
0.2 & -0.1 \\
0.1 & -0.3
\end{array}\right), \quad W(2)=\left(\begin{array}{cc}
0.2 & 0 \\
0.1 & -0.2
\end{array}\right), \\
& H(1)=\left(\begin{array}{cc}
0.2 & 0 \\
0 & 0.1
\end{array}\right), \quad H(2)=\left(\begin{array}{cc}
0.2 & -0.1 \\
0.3 & 0.1
\end{array}\right), \\
& B(1)=\left(\begin{array}{cc}
0 & 1 \\
1 & -1
\end{array}\right), \quad B(2)=\left(\begin{array}{cc}
1 & 0 \\
-1 & 1
\end{array}\right) .
\end{aligned}
$$




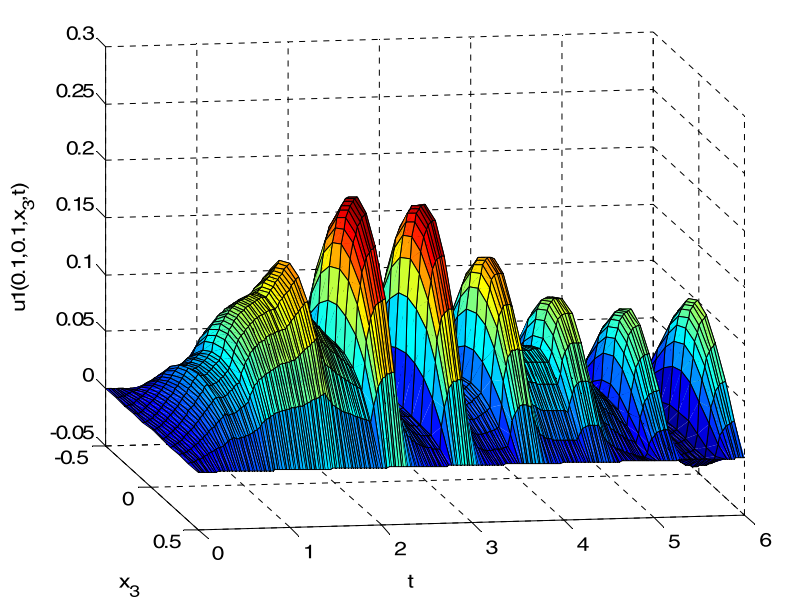

Figure 5 The state surface of $u_{1}\left(0.1,0.1, x_{3}, t\right)$

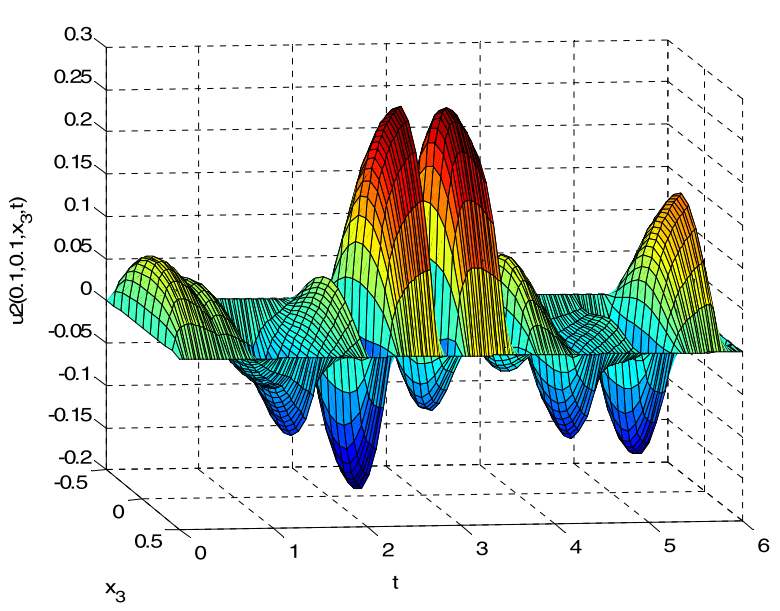

Figure 6 The state surface of $u_{2}\left(0.1,0.1, x_{3}, t\right)$

Figure 7 The Markov switching sequence

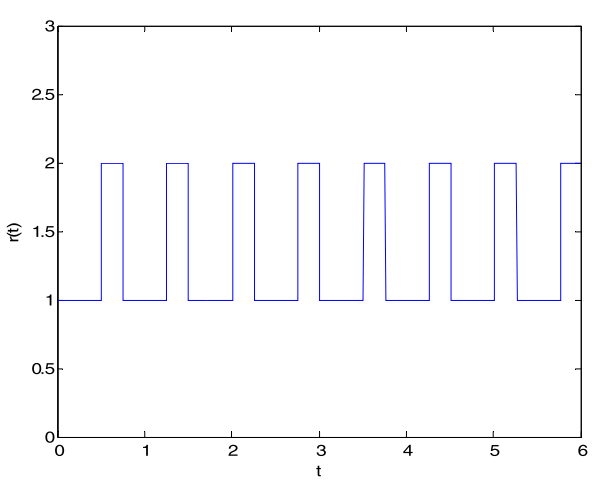

Then a simple computation yields

$$
-\Xi-2 a_{1}(1)+2\left|w_{11}(1)\right| L_{1}+\sum_{j=1, j \neq i}^{n}\left|w_{1 j}(1)\right|+\sum_{j=1, j \neq i}^{n} \frac{q_{j}(1)}{q_{1}(1)}\left|w_{j 1}(1)\right| L_{1}^{2}+\sum_{j=1}^{n}\left|h_{1 j}(1)\right| L_{j}
$$




$$
\begin{aligned}
& +\sum_{j=1}^{n}\left|b_{1 j}(1)\right|+\sum_{j=1}^{n} \frac{q_{j}(1)}{q_{1}(1)}\left|b_{j 1}(1)\right| L_{1}^{2}+\sum_{j=1}^{n} \frac{\left|h_{j 1}(1)\right|}{1-\mu} e^{2 \alpha \tau} \frac{q_{j}(1)}{q_{1}(1)} L_{1}+\sum_{j=1}^{n} \gamma_{1 j} q_{1}(j)+1 \\
= & -1.41<0, \\
-\Xi & -2 a_{2}(1)+2\left|w_{22}(1)\right| L_{2}+\sum_{j=1, j \neq i}^{n}\left|w_{2 j}(1)\right|+\sum_{j=1, j \neq i}^{n} \frac{q_{j}(1)}{q_{2}(1)}\left|w_{j 2}(1)\right| L_{2}^{2}+\sum_{j=1}^{n}\left|h_{2 j}(1)\right| L_{j} \\
& +\sum_{j=1}^{n}\left|b_{2 j}(1)\right|+\sum_{j=1}^{n} \frac{q_{j}(1)}{q_{2}(1)}\left|b_{j 2}(1)\right| L_{2}^{2}+\sum_{j=1}^{n} \frac{\left|h_{j 2}(1)\right|}{1-\mu} e^{2 \alpha \tau} \frac{q_{j}(1)}{q_{2}(1)} L_{2}+\sum_{j=1}^{n} \gamma_{2 j} q_{2}(j)+1 \\
= & -0.71<0 . \\
-\Xi & -2 a_{1}(2)+2\left|w_{11}(2)\right| L_{1}+\sum_{j=1, j \neq i}^{n}\left|w_{1 j}(2)\right|+\sum_{j=1, j \neq i}^{n} \frac{q_{j}(2)}{q_{1}(2)}\left|w_{j 1}(2)\right| L_{1}^{2}+\sum_{j=1}^{n}\left|h_{1 j}(2)\right| L_{j} \\
& +\sum_{j=1}^{n}\left|b_{1 j}(2)\right|+\sum_{j=1}^{n} \frac{q_{j}(2)}{q_{1}(2)}\left|b_{j 1}(2)\right| L_{1}^{2}+\sum_{j=1}^{n} \frac{\left|h_{j 1}(2)\right|}{1-\mu} e^{2 \alpha \tau} \frac{q_{j}(2)}{q_{1}(2)} L_{1}+\sum_{j=1}^{n} \gamma_{1 j} q_{1}(j)+1 \\
= & -0.51<0, \\
-\Xi & -2 a_{2}(2)+2\left|w_{22}(2)\right| L_{2}+\sum_{j=1, j \neq i}^{n}\left|w_{2 j}(2)\right|+\sum_{j=1, j \neq i}^{n} \frac{q_{j}(2)}{q_{2}(2)}\left|w_{j 2}(2)\right| L_{2}^{2}+\sum_{j=1}^{n}\left|h_{2 j}(2)\right| L_{j} \\
& +\sum_{j=1}^{n}\left|b_{2 j}(2)\right|+\sum_{j=1}^{n} \frac{q_{j}(2)}{q_{2}(2)}\left|b_{j 2}(2)\right| L_{2}^{2}+\sum_{j=1}^{n} \frac{\left|h_{j 2}(2)\right|}{1-\mu} e^{2 \alpha \tau} \frac{q_{j}(2)}{q_{2}(2)} L_{2}+\sum_{j=1}^{n} \gamma_{2 j} q_{2}(j)+1 \\
= & -0.91<0 .
\end{aligned}
$$

According to Theorem 1, we can say that system (25) is almost sure ISS. Figures 8 and 9 show that the states of RDNNs (25) in the presence of input and illustrate the feature of ISS that its behavior remains bounded when its inputs are bounded for RDNNs (25). The evolutions of states are shown in Figs. 8 and 9, which also demonstrate that system (25) is almost sure ISS. Figures 10 and 11 show the states of RDNNs (25) in the absence of input. Therefore we can see that these RDNNs have bounded input-bounded output stability and exponential stability.

Figure 8 The transient state of $u_{1}(x, t)$ for RDNNs (25) in presence of input $v(t)$

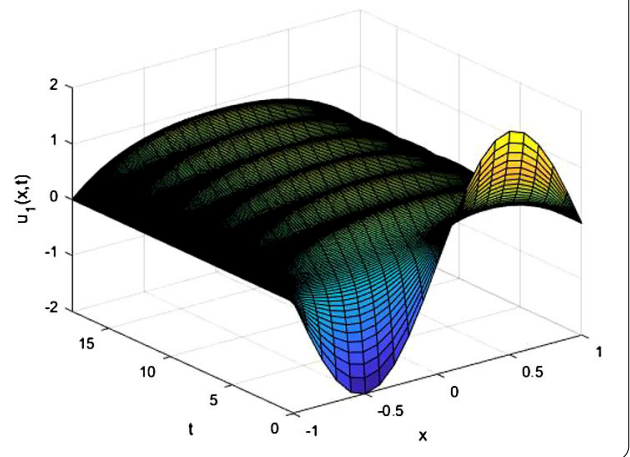


Figure 9 The transient state of $u_{2}(x, t)$ for RDNNs (25) in presence of input $v(t)$

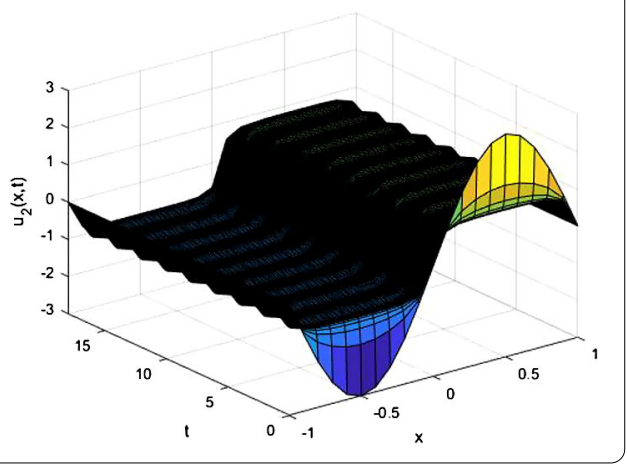

Figure 10 The transient state of $u_{1}(x, t)$ for RDNNs (25) in absence of input $v(t)$

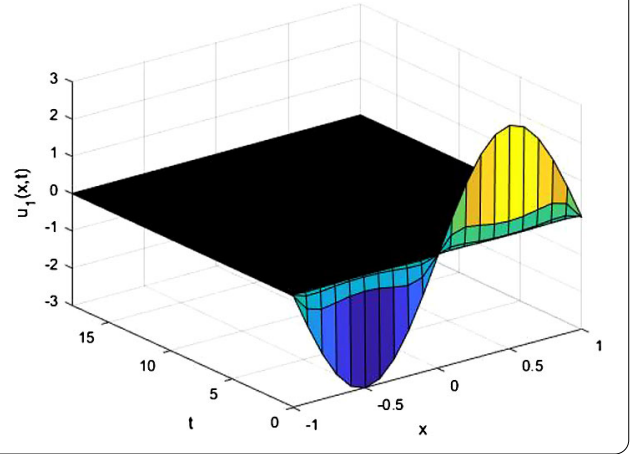

Figure 11 The transient state of $u_{2}(x, t)$ for RDNNs (25) in absence of input $v(t)$

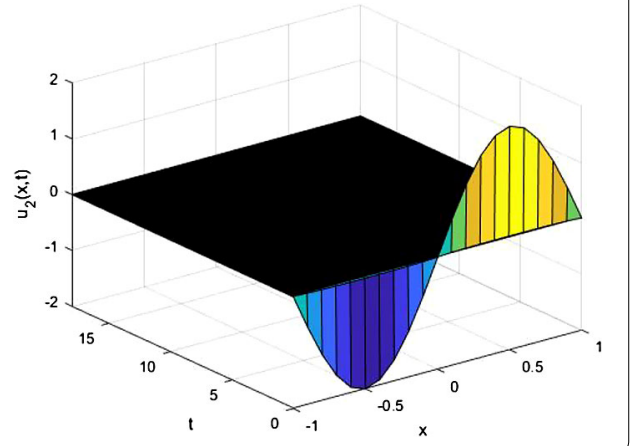

\section{Conclusions}

In this paper, we have considered the almost sure ISS problem for Markovian jump delayed NNs with reaction-diffusion terms and Dirichlet boundary conditions. Sufficient criteria have been obtained guaranteeing almost sure ISS of the considered systems. Different inequalities are employed to deal with the reaction-diffusion terms, and consequently we are convinced that the diffusion do contribute to the ISS analysis of RDNNs. The wellknown Hardy-Poincaré inequality in partial differential equation is used firstly in the ISS problem. Two numerical examples have demonstrated the effectiveness of the proposed approach. 


\section{Funding}

This work is partially supported by the National Natural Science Foundation of China under Grants No. 61573013 and the Special research projects in Shaanxi Province Department of Education under Grant No. 17JK0824.

\section{Competing interests}

The authors declare that they have no competing interests.

\section{Authors' contributions}

The authors declare that the study was realized in collaboration with the same responsibility. Both authors read and approved the final manuscript.

\section{Author details}

${ }^{1}$ Institute of Nonlinear Science, Xianyang Normal University, Xianyang, P.R. China. ${ }^{2}$ School of Science, Xidian University, Xi'an, P.R. China.

\section{Publisher's Note}

Springer Nature remains neutral with regard to jurisdictional claims in published maps and institutional affiliations.

\section{Received: 5 November 2017 Accepted: 6 June 2018 Published online: 20 July 2018}

\section{References}

1. Kwon, O.M., Park, J.H.: Exponential stability analysis for uncertain neural networks with interval time-varying delays. Appl. Math. Comput. 212, 530-541 (2009)

2. Yang, X., Cao, J.: Exponential synchronization of delayed neural networks with discontinuous activations. IEEE Trans. Circuits Syst. I 60(9), 2431-2439 (2013)

3. Huang, C.X., Cao, J.D.: Convergence dynamics of stochastic Cohen-Grossberg neural networks with unbounded distributed delays. IEEE Trans. Neural Netw. 22, 561-572 (2011)

4. Sontag, E.D.: Smooth stabilization implies coprime factorization. IEEE Trans. Autom. Control 34, 435-443 (1989)

5. Sontag, E.D., Wang, Y.: New characterizations of input-to-state stability. IEEE Trans. Autom. Control 41, 1283-1294 (1996)

6. Jiang, Z.P., Wang, Y.: Input-to-state stability for discrete-time nonlinear systems. Automatica 37(6), 857-869 (2001)

7. Hong, Y.G., Jiang, Z.P., Feng, G.: Finite-time input-to-state stability and applications to finite-time control design. SIAM J. Control Optim. 48(7), 4395-4418 (2010)

8. Sontag, E.D.: On the input-to-state stability property. Eur. J. Control 1(1), 24-36 (1995)

9. Sontag, E.D., Wang, Y.: On characterizations of the input-to-state stability property. Syst. Control Lett. 24(5), 351-359 (1995)

10. Dashkovskiy, S., Mironchenko, A.: Input-to-state stability of nonlinear impulsive systems. SIAM J. Control Optim. 51(3), 1962-1987 (2012)

11. Dashkovskiy, S., Ruffer, B., Wirth, F.: Small gain theorems for large scale systems and construction of ISS Lyapunov functions. SIAM J. Control Optim. 48(6), 4089-4118 (2010)

12. Freeman, R.A., Kokotovic, P.V.: Robust Nonlinear Control Design: State-Space and Lyapunov Techniques. Birkhäuser, Boston (2008)

13. Sanchez, E.N., Perez, J.P.: Input-to-state stability (ISS) analysis for dynamic neural networks. IEEE Trans. Circuits Syst. I 46, 1395-1398 (1999)

14. Ahn, C.K.: Passive learning and input-to-state stability of switched Hopfield neural networks with time-delay. Inf. Sci. $180,4582-4594(2010)$

15. Zhu, S., Shen, Y.: Two algebraic criteria for input-to-state stability of recurrent neural networks with time-varying delays. Neural Comput. Appl. 22, 1163-1169 (2013). https://doi.org/10.1007/s00521-012-0882-9

16. Ahn, C.K.: Robust stability of recurrent neural networks with ISS learning algorithm. Nonlinear Dyn. 65, 413-419 (2011)

17. Yang, Z., Zhou, W., Huang, T.: Exponential input-to-state stability of recurrent neural networks with multiple time-varying delays. Cogn. Neurodyn. 8, 47-54 (2014). https://doi.org/10.1007/s11571-013-9258-9

18. Serrano-Gotarredona, T., Linares-Barranco, B.: Log-domain implementation of complex dynamics reaction-diffusion neural networks. IEEE Trans. Neural Netw. 14, 1337-1355 (2003)

19. Lu, J.G.: Robust global exponential stability for interval reaction-diffusion Hopfield neural networks with distributed delays. IEEE Trans. Circuits Syst. II 54, 1115-1119 (2007)

20. Zhang, W., Li, J., Xing, K., Ding, C.: Synchronization for distributed parameter NNs with mixed delays via sampled-data control. Neurocomputing 175, 265-277 (2016)

21. Wang, Z.S., Zhang, H.G., Li, P.: An LMI approach to stability analysis of reaction-diffusion Cohen-Grossberg neural networks concerning Dirichlet boundary conditions and distributed delays. IEEE Trans. Syst. Man Cybern. B 40, 1596-1606 (2010)

22. Wang, Z., Zhang, H.: Global asymptotic stability of reaction-diffusion Cohen-Grossberg neural network with continuously distributed delays. IEEE Trans. Neural Netw. 21, 39-49 (2010)

23. Lu, J.G., Lu, L.J.: Global exponential stability and periodicity of reaction-diffusion recurrent neural networks with distributed delays and Dirichlet boundary conditions. Chaos Solitons Fractals 39, 1538-1549 (2009)

24. Zhang, W., Li, J., Ding, C., Xing, K.: pth moment exponential stability of hybrid delayed reaction-diffusion Cohen-Grossberg neural networks. Neural Process. Lett. 46, 83-111 (2017)

25. Zhang, W.: Passivity analysis of spatially and temporally BAM neural networks with the Neumann boundary conditions. Bound. Value Probl. 2015, Article ID 174 (2015). https://doi.org/10.1186/s13661-015-0435-0

26. Balasubramaniam, P., Vidhya, C.: Exponential stability of stochastic reaction-diffusion uncertain fuzzy neural networks with mixed delays and Markovian jumping parameters. Expert Syst. Appl. 39, 3109-3115 (2012) 
27. Zhang, X., Wu, S., Li, K.: Delay-dependent exponential stability for impulsive Cohen-Grossberg neural networks with time-varying delays and reaction-diffusion terms. Commun. Nonlinear Sci. Numer. Simul. 16, 1524-1532 (2011)

28. Zhang, W., Li, J., Chen, M.: Pinning adaptive synchronization analysis of linearly coupled delayed RDNNs with unknown time-varying coupling strengths. Adv. Differ. Equ. 2014, Article ID 146 (2014)

29. Yang, X., Cao, J., Yang, Z.: Synchronization of coupled reaction-diffusion neural networks with time-varying delays via pinning-impulsive controller. SIAM J. Control Optim. 51(5), 3486-3510 (2013)

30. Rakkiyappan, R., Dharani, S., Zhu, Q.: Synchronization of reaction-diffusion neural networks with time-varying delays via stochastic sampled-data controller. Nonlinear Dyn. 79(1), 485-500 (2015)

31. Rakkiyappan, R., Dharani, S.: Sampled-data synchronization of randomly coupled reaction-diffusion neural networks with Markovian jumping and mixed delays using multiple integral approach. Neural Comput. Appl. 28(3), 449-462 (2017)

32. Schiaffino, A., Tesei, A.: Competition systems with Dirichlet boundary conditions. J. Math. Biol. 15, 93-105 (1982)

33. Mao, X.: Exponential Stability of Stochastic Differential Equations. Marcel Dekker, New York (1994)

34. Luo, Q., Zhang, Y.: Almost sure exponential stability of stochastic reaction diffusion systems. Nonlinear Anal. 71 e487-e493 (2009)

35. Rakkiyappan, R., Zhu, Q., Chandrasekar, A.: Stability of stochastic neural networks of neutral type with Markovian jumping parameters: a delay-fractioning approach. J. Franklin Inst. 351(3), 1553-1570 (2014)

36. Ali, M.S., Gunasekaran, N., Zhu, Q.: State estimation of T-S fuzzy delayed neural networks with Markovian jumping parameters using sampled-data control. Fuzzy Sets Syst. 306, 87-104 (2017)

37. Wang, G., Li, Z., Zhang, Q., Yang, C.: Robust finite-time stability and stabilization of uncertain Markovian jump systems with time-varying delay. Appl. Math. Comput. 293, 377-393 (2017)

38. Shi, G., Ma, Q.: Synchronization of stochastic Markovian jump neural networks with reaction-diffusion terms. Neurocomputing 77, 275-280 (2012)

39. Brezis, H., Vazquez, J.L.: Blow-up solutions of some nonlinear elliptic problem. Rev. Mat. Univ. Complut. Madr. 10, 443-469 (1997)

40. Lou, X., Cui, B.: Stochastic stability analysis for delayed neural networks of neutral type with Markovian jump parameters. Chaos Solitons Fractals 39(5), 2188-2197 (2009)

41. Zhang, Y., Luo, Q.: Global exponential stability of impulsive delayed reaction-diffusion neural networks via Hardy-Poincaré inequality. Neurocomputing 83, 198-204 (2012)

\section{Submit your manuscript to a SpringerOpen ${ }^{\circ}$ journal and benefit from:}

- Convenient online submission

- Rigorous peer review

- Open access: articles freely available online

- High visibility within the field

- Retaining the copyright to your article

Submit your next manuscript at $\gg$ springeropen.com 